\title{
Note on the strong convergence of a weighted sum
}

\author{
Xiaochun Li, Yu Miao and Xiaoman Zhang
}

${ }^{\text {*Correspondence: }}$

L.xiaochun@tom.com

College of Mathematics and

Information Science, Henan Normal

University, Xin Xiang, China

\begin{abstract}
In this paper, we consider an interesting weighted sums $\mu_{n}:=\frac{1}{(\log n)^{1-p}} \sum_{j=2}^{n} \frac{x_{n+2-j}}{(\log )^{p}}$,

$0<p<1$, where $\left\{X, X_{n}, n \geq 1\right\}$ is a sequence of independent and identically

distributed random variables with $\mathbb{E} X=0$, and the equivalence of the almost sure and complete convergence of $\mu_{n}$ is proved.
\end{abstract}

Keywords: strong convergence; complete convergence; weighted sums

\section{Introduction}

Hsu and Robbins [1] introduced the concept of complete convergence as follows: a sequence $\left\{Y_{n}, n \geq 1\right\}$ converges completely to the constant $c$ if

$$
\sum_{n=1}^{\infty} \mathbb{P}\left(\left|Y_{n}-c\right|>\varepsilon\right)<\infty \quad \text { for all } \varepsilon>0 \text {. }
$$

They also show that the sequence of arithmetic means of i.i.d. random variables converges completely to the expected value if the variance of the summands is finite. The converse was proved by Erdös $[2,3]$. Furthermore, if $Y_{n} \rightarrow c$ completely, then the Borel-Cantelli lemma trivially implies that $Y_{n} \stackrel{\text { a.s. }}{\rightarrow} c$, almost sure convergence as $n \rightarrow \infty$. The converse statement is generally, or even 'typically', not true.

There are numerous publications in the literature studying the almost sure convergence and complete convergence for the weighted sums of a sequence of random variables. Gut [4] provided necessary and sufficient conditions for the complete convergence of the Cesáro means of i.i.d. random variables. Li et al. [5] obtained some results on complete convergence for weighted sums of independent random variables. Cuzick [6] proved a strong law for weighted sums of i.i.d. random variables. Miao and Xu [7] established a general result for the weighted sums of stationary sequence.

Throughout this paper, assume that $\left\{X, X_{n}, n \geq 1\right\}$ is a sequence of independent and identically distributed random variables with $\mathbb{E} X=0$. Chow and Lai [8] established the following result.

Theorem 1.1 [8, Theorem 3] Let $X, X_{1}, X_{2}, \ldots$ be i.i.d.random variables such that $\mathbb{E} X_{1}=0$. For $\alpha>1$, the following statements are equivalent:

1. $\mathbb{E} \exp \left(t|X|^{\alpha}\right)<\infty \forall t>0$;

2. $\lim _{n \rightarrow \infty}(\log n)^{-1 / \alpha} X_{n}=0$ a.e.;

3. $\lim _{n \rightarrow \infty}(\log n)^{-1 / \alpha} \sum_{i=1}^{n} c_{n-i} X_{i}=0$ a.e. for some (or equivalently for every) nonvoid sequence of real numbers $\left(c_{n}, n \geq 0\right)$ such that $c_{n}=O\left(n^{-\beta}\right)$, where $\beta=\alpha /(\alpha+1)$. 
As an interesting particular case of Theorem 1.1, we consider the following weighted sums:

$$
\mu_{n}:=\frac{1}{(\log n)^{1-p}} \sum_{j=2}^{n} \frac{X_{n+2-j}}{j(\log j)^{p}}, \quad 0<p<1 .
$$

The aim of this paper is to prove the following.

Theorem 1.2 We have $\mu_{n} \stackrel{\text { a.s. }}{\rightarrow} 0$ if and only if $\mu_{n} \rightarrow 0$ completely.

Remark 1.1 For the case $p=1$, Wu [9] discussed the following weighted sums:

$$
v_{n}:=\frac{1}{\log \log n} \sum_{j=2}^{n} \frac{X_{n+2-j}}{j \log j}
$$

and proved the equivalence of the almost sure and complete convergence of the sequence $v_{n}$. On the one hand, because of the limitation of $\alpha$ in Theorem 1.1, we here only discuss the case of $p>0$. On the other hand, in order to prove the equivalence of the almost sure and complete convergence of $\mu_{n}$ for the case $p=0$, we need the exponential integrability of $X$, but from Theorem 1.1, this does not hold.

Let $S_{j}=\sum_{k=2}^{j} \frac{X_{k}}{k(\log k)^{p}}$; then we have the following.

Corollary 1.1 If $\mu_{n} \stackrel{\text { a.s. }}{\rightarrow} 0$, then

$$
\frac{1}{(\log n)^{1-p}} \max _{2 \leq j \leq n} S_{j} \rightarrow 0 \quad \text { completely. }
$$

\section{Proofs of main results}

Let $[\cdot]$ denote the usual integer part of ' ' and assume that $n>n_{0}=\left[e^{20}\right]$. The constant $C$ in the proofs below depends only on the distribution of the underlying random variable $X$ and may denote different quantities at different appearances.

\subsection{Proof of Theorem 1.2}

The proof of Theorem 1.2 can be derived from Lemma 2.2 and Lemma 2.3.

Lemma 2.1 The following estimate holds:

$$
\sum_{j=\left[\frac{1}{2} \log n\right]}^{n} \log \left(1+C\left(\frac{\log n}{j(\log j)^{p}}\right)^{2}\right)<C \log n
$$

Proof Since $g(j)=\log \left(1+C\left(\frac{\log n}{j(\log j)^{p}}\right)^{2}\right)$ decreases in $j$,

$$
\begin{aligned}
& \sum_{j=\left[\frac{1}{2} \log n\right]}^{n} \log \left(1+C\left(\frac{\log n}{j(\log j)^{p}}\right)^{2}\right) \\
& \leq \int_{\frac{1}{2} \log n}^{n} \log \left(1+C\left(\frac{\log n}{x(\log x)^{p}}\right)^{2}\right) d x+O(1)
\end{aligned}
$$




$$
\begin{aligned}
& \leq \log n \int_{\frac{1}{2}}^{\frac{n}{\log n}} \log \left(1+C\left(\frac{1}{t(\log t+\log \log n)^{p}}\right)^{2}\right) d t+O(1) \\
& \leq \log n \int_{\frac{1}{2}}^{\infty} \log \left(1+C\left(\frac{1}{t(\log t+\log \log n)^{p}}\right)^{2}\right) d t+O(1) \\
& \leq C \log n
\end{aligned}
$$

which yields the desired result.

Lemma 2.2 If $\mu_{n} \stackrel{\text { a.s. }}{\longrightarrow} 0$, then

$$
\mu_{n}^{(1)}:=\frac{1}{(\log n)^{1-p}} \sum_{j=\left[\frac{1}{2} \log n\right]}^{n} \frac{X_{n+2-j}}{j(\log j)^{p}} \rightarrow 0
$$

completely and

$$
\frac{1}{(\log n)^{1-p}} \sum_{j=\left[\frac{1}{2} \log n\right]+1}^{\left[\frac{1}{2} \log [n \log n]\right]} \frac{X_{n+2-j}}{j(\log j)^{p}} \rightarrow 0
$$

completely.

Proof From Theorem 1.1, we know $\mathbb{E} e^{|X|^{\frac{1}{1-p}}}<\infty$, which implies $\mathbb{E} e^{|X|}<\infty$. Hence by the elementary inequality

$$
\left|e^{\eta x}-1-\eta x\right| \leq \eta^{2} e^{|x|} \quad \text { for all } \eta \in[0,1]
$$

we have

$$
\mathbb{E} e^{\eta X} \leq 1+\eta^{2} \mathbb{E} e^{|X|}=1+\eta^{2} C
$$

Notice that if $n>n_{0}$ and $n \geq j \geq\left[\frac{1}{2} \log n\right]$, then $\frac{\log n}{j(\log j)^{p}}<1$. Hence, by Lemma 2.1, for any $\varepsilon>0$,

$$
\begin{aligned}
\sum_{n=n_{0}}^{\infty} \mathbb{P}\left(\mu_{n}^{(1)}>\varepsilon\right) & \leq \sum_{n=n_{0}}^{\infty} n^{-\varepsilon(\log n)^{(1-p)}} \mathbb{E} e^{\log n \sum_{j=\left[\frac{1}{2} \log n\right]}^{\frac{X_{n+2-j}}{j(\log j)^{p}}}} \\
& =\sum_{n=n_{0}}^{\infty} n^{-\varepsilon(\log n)^{(1-p)}} \prod_{j=\left[\frac{1}{2} \log n\right]}^{n} \mathbb{E} e^{\frac{\log n}{(\log j)^{p}} X_{n+2-j}} \\
& \leq \sum_{n=n_{0}}^{\infty} n^{-\varepsilon(\log n)^{(1-p)}} \prod_{j=\left[\frac{1}{2} \log n\right]}^{n}\left(1+C\left(\frac{\log n}{j(\log j)^{p}}\right)^{2}\right) \\
& \leq \sum_{n=n_{0}}^{\infty} n^{-\varepsilon(\log n)^{(1-p)}} e^{C \log n} \\
& <\infty
\end{aligned}
$$


Similarly, we can obtain $\sum_{n=n_{0}}^{\infty} \mathbb{P}\left(\mu_{n}^{(1)}<-\varepsilon\right)<\infty$, then $\sum_{n=n_{0}}^{\infty} \mathbb{P}\left(\left|\mu_{n}^{(1)}\right|>\varepsilon\right)<\infty$. The first statement now follows if we combine the two inequalities. The same technique yields the second statement.

Lemma 2.3 If

$$
\mu_{n}^{(2)}:=\frac{1}{(\log n)^{1-p}} \sum_{j=2}^{\left[\frac{1}{2} \log n\right]} \frac{X_{n+2-j}}{j(\log j)^{p}} \stackrel{\text { a.s. }}{\rightarrow} 0,
$$

then $\mu_{n}^{(2)} \rightarrow 0$ completely.

Proof For any $\varepsilon>0$ we have

$$
0=\mathbb{P}\left(\limsup _{n \rightarrow \infty}\left|\mu_{n}^{(2)}\right|>\varepsilon\right) \geq \mathbb{P}\left(\limsup _{m \rightarrow \infty}\left|\mu_{n(m)}^{(2)}\right|>\varepsilon\right),
$$

where $n(m)=[m \log m], m \in \mathbb{N}$. Since

$$
n(m+1)+2-\frac{1}{2} \log n(m+1)>n(m)
$$

for $m>3$, the random variables $\mu_{n(m)}^{(2)}, m=4,5, \ldots$ are independent. Notice also that $(\log n(m))^{1-p}<(2 \log m)^{1-p}$. So by the Borel-Cantelli lemma and the second statement of Lemma 2.2, we have $\sum_{m=n_{0}}^{\infty} \mathbb{P}\left(\left|\mu_{n(m)}^{(2)}\right|>\varepsilon\right)<\infty$, proving the lemma.

\subsection{Proof of Corollary 1.1}

We easily see that

$$
\mathbb{E} S_{n}^{2}=\mathbb{E} X^{2} \sum_{j=2}^{n} \frac{1}{\left(j(\log j)^{p}\right)^{2}}<C<\infty .
$$

Now we apply the inequality

$$
\mathbb{P}\left(\max _{2 \leq j \leq n} S_{j}>\eta\right) \leq 2 \mathbb{P}\left(S_{n}>\eta-\sqrt{\mathbb{E} S_{n}^{2}}\right)
$$

(cf. Chow and Teicher [10], p.111) by taking $\eta=(\log n)^{1-p} \varepsilon$ for $\varepsilon>0$. Since $\eta-\sqrt{\mathbb{E} S_{n}^{2}}>\frac{1}{2} \eta$ for all $n$ sufficiently large,

$$
\mathbb{P}\left(\max _{2 \leq j \leq n} \frac{S_{j}}{(\log n)^{1-p}}>\varepsilon\right) \leq 2 \mathbb{P}\left(\frac{1}{(\log n)^{1-p}} \sum_{j=2}^{n} \frac{X_{n+2-j}}{j(\log j)^{p}} \geq \frac{1}{2} \varepsilon\right) .
$$

Therefore,

$$
\sum_{n=n_{0}}^{\infty} \mathbb{P}\left(\max _{2 \leq j \leq n} \frac{S_{j}}{(\log n)^{1-p}}>\varepsilon\right) \leq 2 \sum_{n=n_{0}}^{\infty} \mathbb{P}\left(\mu_{n}>\frac{1}{2} \varepsilon\right)<\infty .
$$

Replacing $X_{j}$ with $-X_{j}$, the corollary follows. 
Competing interests

The authors declare that they have no competing interests.

\section{Authors' contributions}

All authors contributed equally to the manuscript, read and approved the final manuscript.

\section{Acknowledgements}

This work is supported by HASTIT (No. 2011 HASTIT011), NSFC (No. 11001077), NCET (NCET-11-0945), and Plan For

Scientific Innovation Talent of Henan Province (124100510014).

Received: 11 January 2014 Accepted: 21 April 2014 Published: 12 May 2014

\section{References}

1. Hsu, PL, Robbins, H: Complete convergence and the law of large number. Proc. Natl. Acad. Sci. USA 33, 25-31 (1947)

2. Erdös, P: On a theorem of Hsu and Robbins. Ann. Math. Stat. 20, 286-291 (1949)

3. Erdös, P: Remark on my paper 'On a theorem of Hsu and Robbins'. Ann. Math. Stat. 21, 138 (1950)

4. Gut, A: Complete convergence and Cesáro summation for i.i.d. random variables. Probab. Theory Relat. Fields 97 , 169-178 (1993)

5. Li, DL, Rao, MB, Jiang, TF, Wang, XC: Complete convergence and almost sure convergence of weighted sums of random variables. J. Theor. Probab. 8, 49-76 (1995)

6. Cuzick, J: A strong law for weighted sums of i.i.d. random variables. J. Theor. Probab. 8, 625-641 (1995)

7. Miao, Y, Xu, SF: Almost sure convergence of weighted sum. Miskolc Math. Notes 14,173-181 (2013)

8. Chow, YS, Lai, TL: Limiting behavior of weighted sums of independent random variables. Ann. Probab. 1, 810-824 (1973)

9. Wu, WB: On the strong convergence of a weighted sum. Stat. Probab. Lett. 1, 19-22 (1999)

10. Chow, YS, Teicher, H: Probability Theory, 3rd edn. Springer, New York (1978)

10.1186/1029-242X-2014-179

Cite this article as: Li et al.: Note on the strong convergence of a weighted sum. Journal of Inequalities and Applications 2014, 2014:179

\section{Submit your manuscript to a SpringerOpen ${ }^{\ominus}$ journal and benefit from:}

- Convenient online submission

- Rigorous peer review

Immediate publication on acceptance

- Open access: articles freely available online

- High visibility within the field

- Retaining the copyright to your article 